\title{
Nonlinear quasi-steady state benchmark of global gyrokinetic codes
}

\author{
X. Lapillonne, ${ }^{1}$ B. F. McMillan, ${ }^{1}$ T. Görler, ${ }^{2}$ S. Brunner, ${ }^{1}$ T. Dannert, ${ }^{2}$ F. Jenko, ${ }^{2}$ F. \\ Merz, ${ }^{2}$ and L. Villard ${ }^{1}$ \\ 1) Ecole Polytechnique Fédérale de Lausanne (EPFL), Centre de Recherches \\ en Physique des Plasmas, Association Euratom-Confédération Suisse, \\ CH-1015 Lausanne, Switzerland \\ 2) Max-Planck-Institut für Plasmaphysik, Boltzmannstr. 2, D-85748 Garching, \\ Germany
}

Two global gyrokinetic codes are benchmarked against each other by comparing simulation results in the case of ion temperature gradient driven (ITG) turbulence, in the adiabatic electron response limit. The two codes are the Eulerian code GENE and the Lagrangian Particle-In-Cell code ORB5 which solve the gyrokinetic equations. Linear results are presented, including growth rates, real frequencies and mode structure comparisons. Nonlinear simulations without sources are carried out with particular attention to considering the same initial conditions, showing identical linear phase and first nonlinear burst. Very good agreement is also achieved between simulations obtained using a Krook-type heat source, which enables to reach a quasi-steady state and thus to compare the heat diffusivity traces over a statistically meaningful time interval. For these nonlinear results, the radial zonal flow structure and shearing rate profile are also discussed. The very detailed comparisons presented may serve as reference for benchmarking other gyrokinetic simulation codes, in particular those which consider global geometry. 


\section{INTRODUCTION}

In fusion research devices based on magnetic confinement, such as Tokamaks, the energy and particle transport is significantly larger than expected from purely collisional processes. This anomalous transport is commonly attributed to small-scale turbulence, generated by microinstabilities, which are driven by temperature and density gradients. A better understanding of these microinstabilities and associated turbulence is therefore of key importance in view of achieving nuclear fusion and they are actively investigated in the frame of the gyrokinetic theory ${ }^{1,2}$ by means of numerical simulations, see Garbet et al. ${ }^{3}$ for a recent overview. Among the different physical models that have been considered in the wide variety of codes developed to solve the gyrokinetic equation, we shall in particular mention the local $^{4-8}$ and global ${ }^{9-14}$ approaches. In the local treatment, the so-called flux-tube approach ${ }^{15}$, only a reduced simulation domain corresponding to a small plasma volume aligned with the magnetic field lines is considered and the radial variations of macroscopic quantities such as the density and temperature fields and their gradients as well as of the magnetic field are neglected. This local approach enables to significantly reduce the computational cost of microturbulence simulations. In some cases however, when the characteristic size of the turbulence is not negligible with respect to the machine size, e.g. in a small device, or with respect to a characteristic profile gradient length, such as found in transport barriers, a global approach may be necessary, where the full torus is considered and radial variations of equilibrium quantities are retained. Besides these two approximations of the physical system, different numerical methods and implementations are also used in the various codes, and important tests and benchmarks are thus required for their validation. To this end, several efforts have been carried out in the past years ${ }^{16-19}$, mainly focussing on linear growth rates and nonlinear heat diffusivity comparisons. A very good level of agreement was in particular reached over statistically significant time intervals for the heat diffusivity computed with nonlinear flux-tube simulations ${ }^{17-19}$. Concerning the global approach, a qualitative and semi-quantitative agreement between different codes was for instance reached in the last publication ${ }^{19}$. However, these nonlinear global simulations were considering a problem without source term in which the system relaxes to a marginal state and for which the turbulent transport occurs only transiently and can depend on details in the initial system, making relevant quantitative comparisons difficult. The need for more detailed and accurate 
benchmarking between global gyrokinetic codes thus appears necessary.

In the present work, detailed benchmarks between the global version of the Eulerian code GENE $^{14,20,21}$ and the global Lagrangian PIC code ORB $5^{22,23}$ are presented. In addition to linear growth rates and nonlinear heat diffusivities, a particular emphasis is given to the mode structure analysis. Nonlinear simulations including a Krook-type heat source are also shown, allowing for the first time to perform systematic quantitative comparisons between two global gyrokinetic codes in quasi-steady state over statistically relevant time intervals. The remainder of the present paper, which can be viewed as a contribution to the current emphasis on validation and verification of fusion-relevant simulations ${ }^{24}$, is organized as follows. In section II, the model equations considered in the two codes as well as some details on their numerical implementation are discussed. Linear results are then presented in section III, showing growth rates, real frequencies and mode structure comparisons. This is followed in section IV by the Rosenbluth-Hinton test showing estimates of zonal flow residuals. Comparisons of nonlinear simulations without sources are then presented in section VA. To obtain these results, a particular effort was made for using exactly the same initial conditions, thus allowing for the detailed comparison of the linear phase and first burst. Finally, nonlinear simulations in quasi-steady state obtained thanks to the implementation of source terms, are presented in section VB. In addition to the heat diffusivity time traces, a particular emphasis is given to the zonal flow structures. Conclusions are drawn in section VI.

\section{THE NUMERICAL MODELS}

In gyrokinetic theory, each plasma species is described in terms of its particle distribution function $f$ in a reduced 5 -dimensional phase space $\left(\vec{X}, v_{\|}, \mu\right)$, where $\vec{X}$ is the gyrocenter position, $v_{\|}$the velocity parallel to the magnetic field, and $\mu=\left(m v_{\perp}^{2}\right) /\left(2 B_{0}\right)$ the magnetic moment. Here $m$ and $q$ will respectively stand for the mass and electric charge of each species, while the equilibrium magnetic field is given by $\vec{B}_{0}$. The time evolution of each distribution function $f\left(t, \vec{X}, v_{\|}, \mu\right)$ is described by the gyrokinetic equation ${ }^{2}$ :

$$
\frac{d f}{d t}=\frac{\partial f}{\partial t}+\dot{\vec{X}} \cdot \frac{\partial f}{\partial \vec{X}}+\dot{v}_{\|} \frac{\partial f}{\partial v_{\|}}=0
$$


where the equations of motion for the gyrocenter variables $\left(\vec{X}, v_{\|}, \mu\right)$ read:

$$
\begin{aligned}
& \dot{\vec{X}}=\vec{v}_{G}=v_{\|} \vec{b}_{0}+\frac{B_{0}}{B_{0 \|}^{*}}\left(\vec{v}_{E}+\vec{v}_{\nabla B}+\vec{v}_{c}\right), \\
& \dot{v}_{\|}=-\frac{1}{m v_{\|}} \vec{v}_{G} \cdot\left(q \vec{\nabla} \bar{\phi}+\mu \vec{\nabla} B_{0}\right), \\
& \dot{\mu}=0
\end{aligned}
$$

with $\vec{v}_{E}$ the $\vec{E} \times \vec{B}$ drift velocity

$$
\vec{v}_{E}=-\frac{\vec{\nabla} \bar{\Phi} \times \vec{B}_{0}}{B_{0}^{2}},
$$

where $\bar{\Phi}$ stands for the gyroaveraged electrostatic potential. The grad- $B$ drift velocity is given by

$$
\vec{v}_{\nabla B}=\frac{\mu}{m \Omega B_{0}} \vec{B}_{0} \times \vec{\nabla} B_{0}
$$

and the curvature drift velocity by

$$
\vec{v}_{c}=\frac{\mu_{0} v_{\|}^{2}}{\Omega B_{0}^{2}} \vec{b}_{0} \times \vec{\nabla}\left(p_{0}+\frac{B_{0}^{2}}{2 \mu_{0}}\right) .
$$

One finally defines $B_{0 \|}^{*}=\left[\vec{B}_{0}+(m / q) v_{\|} \vec{\nabla} \times \vec{b}_{0}\right] \cdot \vec{b}_{0}$, where $\vec{b}_{0}$ is the unit vector parallel to the equilibrium magnetic field. Note that although both codes can account for electromagnetic perturbations, we constrain ourselves in this work to studying electrostatic fluctuations, and the present equations have therefore been given in this limit.

In both codes, the particle distribution function $f$ of each species is split into an equilibrium $f_{0}$ and a perturbed part $\delta f, f=f_{0}+\delta f$ assuming $|\delta f| \ll\left|f_{0}\right|$. In GENE, $f_{0}$ is chosen as a local Maxwellian, whereas a canonical Maxwellian is usually considered in ORB $5^{25}$.

The GENE code, which was recently extended from a local to a global version ${ }^{14,20,21}$, considers an Eulerian approach in which the gyrokinetic equation (1) is first discretized on a fixed grid in phase space. The resulting system of ordinary differential equations (ODEs) for the time evolution of the discretized particle distribution function is then numerically integrated. The ORB5 code on the other hand is a Particle-In-Cell (PIC) code, based on a Lagrangian approach, where the plasma is described by a statistical sampling of phase space using so-called marker (or numerical) particles. The method then consists in following the trajectories of these markers in phase-space.

For the different results that shall be presented in the following, a finite aspect ratio, circular concentric flux-surface equilibrium model is used. This model was shown to give very close 
results to those using a numerical low $\beta$ ideal MHD equilibrium with circular boundary ${ }^{26}$. When using this ad-hoc circular model, the pressure term $\vec{\nabla} p_{0}$ appearing in Eq. (7) is set to zero in GENE, while it remains active in ORB5 simulations and is obtained from $\vec{\nabla} p_{0}=\left(1 / \mu_{0}\right)\left(\vec{\nabla} \times \vec{B}_{0}\right) \times \vec{B}_{0}$, using the approximate equilibrium magnetic field. Furthermore, $B_{0 \|}^{*}$ has been approximated by $B_{0}$ in GENE.

In order to close the system, the quasi-neutrality equation is solved for the electrostatic potential $\Phi$ associated with the fluctuation. Assuming an adiabatic electron response, the following equation is solved in GENE:

$$
\begin{aligned}
-\frac{e n_{0 e}}{T_{0 e}}(\Phi & -\langle\Phi\rangle)+\frac{2 \pi Z_{i}}{m_{i}} \int B_{0 \|}^{*} \delta \bar{f} d v_{\|} d \mu \\
- & \frac{Z_{i} q_{i} n_{0 i}}{T_{0 i}}\left[\Phi-\frac{B_{0}}{T_{0 i}} \int \overline{\bar{\Phi}} \exp \left(-\frac{\mu B_{0}}{T_{0 i}}\right) d \mu\right]=0 .
\end{aligned}
$$

where $\delta \bar{f}$ stands for the gyroaveraged fluctuation part of the ion distribution and $\overline{\bar{\Phi}}$ for the double gyroaveraging of $\Phi$. In Eq. (8), the variations of equilibrium quantities with respect to the ion Larmor radius $\rho_{i}$ have been neglected. With respect to the fluctuations, $k_{\perp} \rho_{i}$ terms are retained to all orders in GENE, while only a second order expansion in $k_{\perp} \rho_{i}$ is considered in ORB5. ( $k_{\perp}$ being the wave number perpendicular to the magnetic field). The quasi-neutrality equation in ORB5 is thus given by:

$$
\begin{gathered}
-\frac{e n_{0 e}}{T_{0 e}}(\Phi \\
-\langle\Phi\rangle)+\frac{2 \pi Z_{i}}{m_{i}} \int B_{0 \|}^{*} \delta \bar{f} d v_{\|} d \mu \\
+\nabla_{\perp}\left(\frac{Z_{i} n_{0 i}}{B_{0} \Omega_{i}} \nabla_{\perp} \Phi\right)=0 .
\end{gathered}
$$

Note, that variations of equilibrium quantities with respect to $\rho_{i}$ are retained in Eq. (9). Neglecting them would correspond to taking the $\left(Z_{i} n_{0 i}\right) /\left(B_{0} \Omega_{i}\right)$ factor out of the first $\nabla_{\perp}$ derivative in this equation, which would them become equivalent to (8) in the limit of a second order expansion in $k_{\perp} \rho_{i}$ of $\Phi$.

In order to allow for quasi-steady state nonlinear simulations, a Krook-type heat source $S_{k}$ can be added to the right hand side of the gyrokinetic equation. In the ORB5 code, this term is implemented $\mathrm{as}^{27}$ :

$$
S_{K}(r, \epsilon)=-\gamma_{h}\left[\delta f(r, \epsilon)-f_{0}(r, \epsilon) \frac{\int d \vec{v} \delta f(r, \epsilon)}{\int d \vec{v} f_{0}(r, \epsilon)}\right],
$$

where $\epsilon=m v_{\|}^{2} / 2+B_{0} \mu$ denotes the kinetic energy, $r$ the minor radius of the considered circular flux-surfaces and $\delta f(r, \epsilon)$ the fluctuating component of the distribution with respect 
to these two variables.=. A similar form is used in GENE, given by:

$$
S_{K}\left(r,\left|v_{\|}\right|, \mu\right)=-\gamma_{h}\left[\left\langle\delta f\left(\vec{X},\left|v_{\|}\right|, \mu\right)\right\rangle-\left\langle f_{0}\left(\vec{X},\left|v_{\|}\right|, \mu\right)\right\rangle \frac{\left\langle\int d \vec{v}\left\langle\delta f\left(\vec{X},\left|v_{\|}\right|, \mu\right)\right\rangle\right\rangle}{\left\langle\int d \vec{v}\left\langle f_{0}\left(\vec{X}, v_{\|}, \mu\right)\right\rangle\right\rangle}\right]
$$

where $\langle$.$\rangle refers to the flux-surface average and$

$$
\delta f\left(\vec{X},\left|v_{\|}\right|, \mu\right)=\frac{\delta f\left(\vec{X}, v_{\|}, \mu\right)+\delta f\left(\vec{X},-v_{\|}, \mu\right)}{2} .
$$

Note that (10) differs from (11) as a result of having replaced $\delta f(r, \epsilon)$ with $\left\langle\delta f\left(\vec{X},\left|v_{\|}\right|, \mu\right)\right\rangle$ in the GENE implementation. This was done for practical reasons, as the computation of the distribution $\delta f(r, \epsilon)$ in variables $(r, \epsilon)$ involves an energy binning which is not aligned with the $\left(v_{\|}, \mu\right)$ grid used in GENE, and therefore would involve cumbersome integrations. Although the exact implementations differ, both operators are designed to keep the temperature close to the initial profile considered for $f_{0}$ while conserving the flux-surface averaged density and parallel momentum. The heating rate constant $\gamma_{h}$ is in general chosen to be about ten times smaller than the typical linear growth rate, to ensure that the effect of the heating operator would not strongly affect the turbulence dynamics ${ }^{27}$.

More details concerning the exact numerical implementation may be found in the corresponding ORB5 $5^{22}$ and $\mathrm{GENE}^{14,20,21}$ publications.

In the following, one has used the relation

$$
k_{\theta}=\frac{n q_{0}}{r_{0}}
$$

as a definition for estimating the average poloidal wave number; where $n$ is the toroidal mode number, while $q_{0}$ and $r_{0}$ are respectively the safety factor and minor radius both evaluated at $r=0.5 a, a$ being the outer minor radius of the Tokamak. One shall also make use of the straight field line poloidal angle $\chi$, which is defined such that the magnetic field lines are straight on a given flux surface in the $(\chi, \phi)$ plane, where $\phi$ is the toroidal angle.

\section{LINEAR RESULTS}

For the present benchmark, physical parameters similar to the standard Cyclone Base Case $(\mathrm{CBC})^{16}$ are considered. A magnetic equilibrium with circular concentric flux-surface 


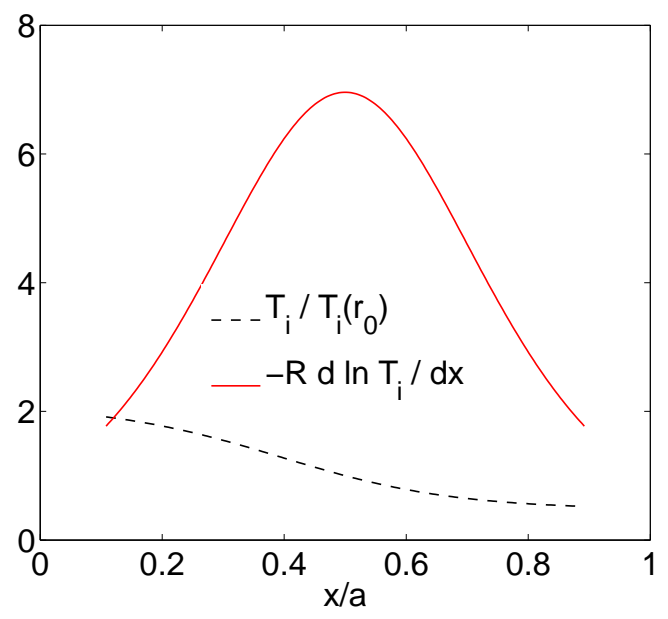

FIG. 1. (Color online) Ion temperature profile and corresponding logarithmic gradient profile, as given by relation (15) with $\kappa_{T_{i}}=6.96$ and $\Delta T_{i}=0.3$.

is used with inverse aspect ration $a / R=0.36$ and safety factor profile:

$$
q(r)=0.86-0.16 r / a+2.52(r / a)^{2},
$$

which corresponds to a local safety factor $q_{0}=1.4$ and shear $\hat{s}=0.8$ at $r=0.5 a$. The ratio between the ion Larmor radius $\rho_{i}$ and minor radius $a$ is taken as $\rho^{*}=\rho_{i} / a=1 / 180$, i.e. consistent with the DIII-D shot that inspired the Cyclone test case. The temperature and density profiles are given by the functional form (where $\mathrm{A}$ stands either for $T_{i}$ or $n$ ):

$$
\hat{A}(r)=\frac{A(r)}{A\left(r_{0}\right)}=\exp \left[-\kappa_{A} a \Delta A \tanh \left(\frac{r-r_{0}}{\Delta A a}\right)\right],
$$

which corresponds to peaked gradient profiles as illustrated in Fig.1. The profile parameters are set to $\kappa_{T i}=6.96, \kappa_{n}=2.23, \Delta T_{i}=\Delta n=0.3$ corresponding to peaked gradient profiles centered at $r_{0}=0.5 \mathrm{a}$. An adiabatic electron response is assumed and the ratio of electron to ion temperature profiles is $T_{e} / T_{i} \equiv 1$.

For these parameters, toroidal Ion Temperature Gradient (ITG) instabilities are the most unstable modes and the corresponding linear growth rates and real frequencies computed with GENE and ORB5 are shown in Figs. 2 and 3 for different mode numbers $k_{\theta}=n q_{0} / r_{0}$. Comparing growth rates obtained with GENE and ORB5, a good agreement is found for the lower $k_{\theta} \rho_{i}$ values, while some discrepancies are observed for $k_{\theta} \rho_{i} \gtrsim 0.3$. Concerning the real frequencies, the two curves agree within 20\%. Among the differences in the model equations 


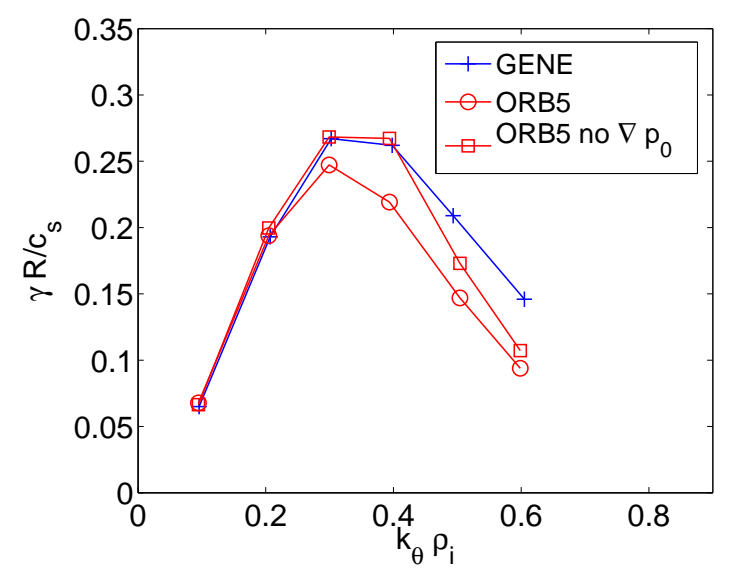

FIG. 2. (Color online) Linear growth rates of toroidal-ITG modes as a function of $k_{\theta} \rho_{i}$ obtained with GENE and ORB5 for CBC-like parameters. The ORB5 results have been obtained using either the standard version of the code (circle), or by setting the $\nabla p_{0}$ contribution to zero.

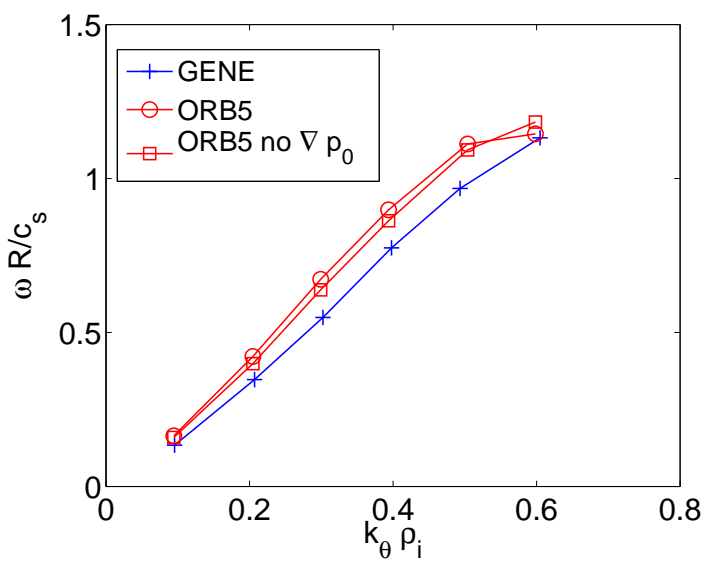

FIG. 3. (Color online) Real frequencies as a function of $k_{\theta} \rho_{i}$ obtained with GENE and ORB5 for CBC-like parameters. Same labels as in Fig. 2.

considered by the two codes, it was found that the $\nabla p_{0}$ term in Eq. (7) for the curvature drift (set to zero in GENE, retained in ORB5) has the largest effect. Much closer growth rates are indeed obtained when setting this contribution to zero in ORB5, as illustrated in Fig. 2. One may be surprised by the strong influence of the $\nabla p_{0}$ term on the linear growth rate, as we are considering only electrostatic perturbations and assuming a low $\beta$ limit equilibrium. It is however important to recall that this pressure correction is obtained 

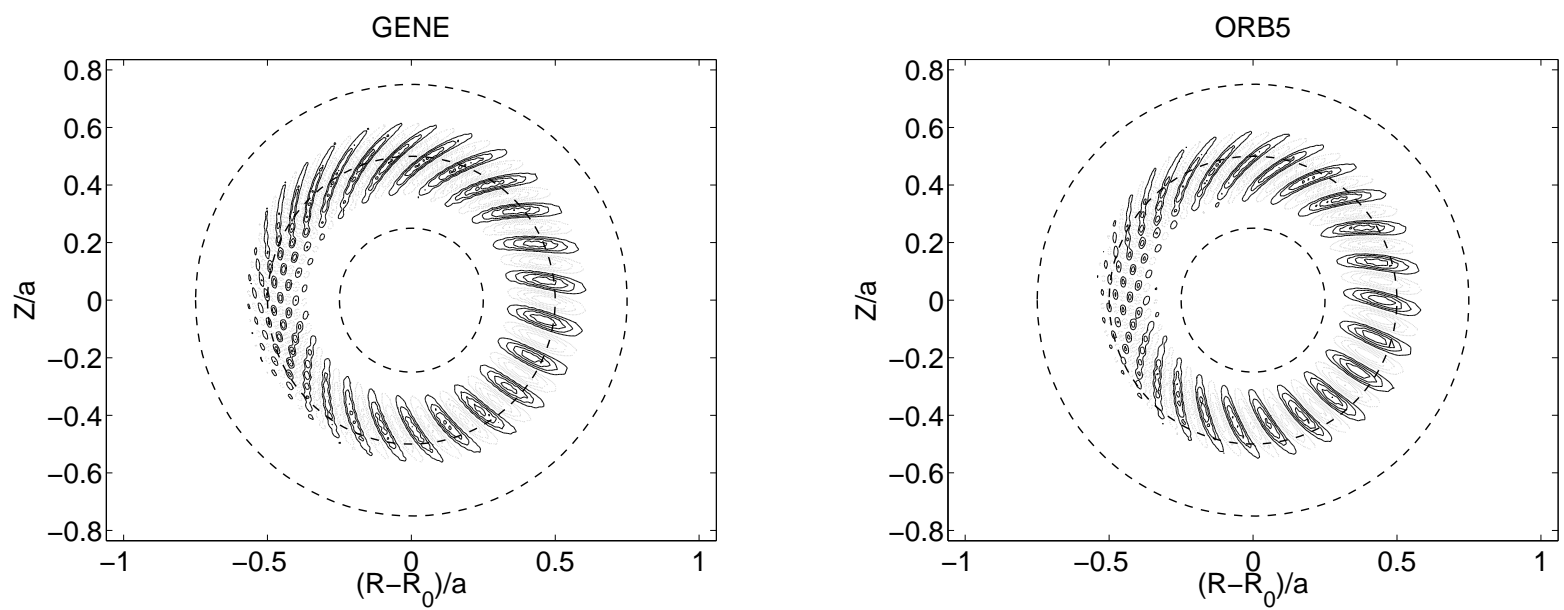

FIG. 4. Contour plots of the electrostatic potential $\Phi$ for mode number $k_{\theta} \rho_{i}=0.3$ for CBC-like parameters, obtained respectively with GENE (left) and ORB5 (right).

here from an ad-hoc equilibrium magnetic field, which is not a true solution of the GradShafranov equation. The $\nabla p_{0}$ contribution might thus be overestimated in comparison to a corresponding low $\beta$ MHD equilibrium. The growth rates for the largest $k_{\theta} \rho_{i}$ are still lower in ORB5, even when setting $\nabla p_{0}$ to zero as compared to GENE. This may result from differences in the field solver as the ORB5 code considers a second order expansion in $k_{\perp} \rho_{i}$ of the polarization density contribution to the quasi-neutrality equation, while GENE keeps all orders in $k_{\perp} \rho_{i}$ of this term, as already mentioned in Sec. II. Concerning the real frequencies, some small deviations remain, which probably result from other minor differences between the two codes.

In addition to these quantitative investigations of linear growth rates and real frequencies, a detailed comparison of the corresponding mode structures was carried out. In Fig. 4, the contour plots of the electrostatic potential $\Phi$ are shown for the toroidal mode number $n=19$, corresponding to the average poloidal wave number $k_{\theta} \rho_{i}=0.3$. Remarkable similarity is observed between results from the two codes. In order to further analyze the mode structures, a comparison of the electrostatic potential as a function of the straight field line poloidal angle $\chi$ is carried out on the given magnetic surface $r=r_{0}=0.5 a$. The considered field $\Phi(r, \chi)$ is obtained in both GENE and ORB5 from a snapshot at the end of the simulation, i.e. when the linear growth rate is converged. As a consequence, the global phases and 


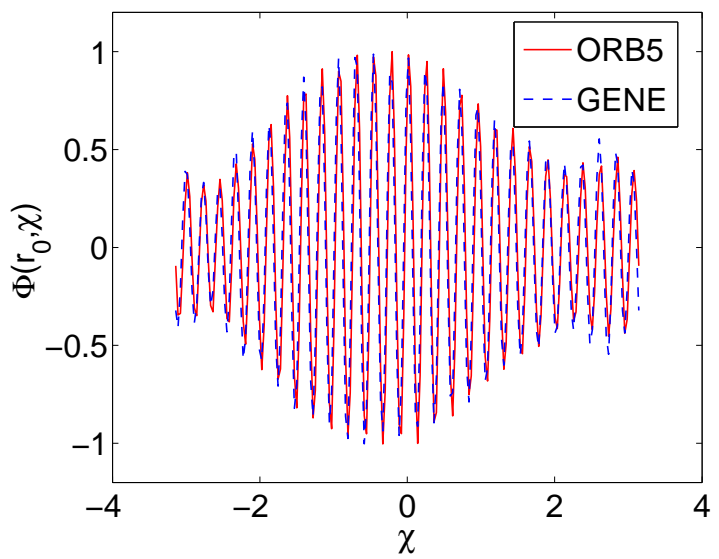

FIG. 5. (Color online) Electrostatic potential at constant $r=0.5 a$ as a function of the straight field line poloidal angle $\chi$ for $k_{\theta} \rho_{i}=0.3$. These plots are obtained after normalizing according to relation (17)

amplitudes of $\Phi$ from the two computations are in general different. In order to be able to compare the potentials from the two codes, the amplitudes and phases of the fields $\Phi\left(r=r_{0}, \chi\right)$ need to be appropriately re-normalized. This is achieved by making use of a poloidal Fourier transform of $\Phi\left(r_{0}, \chi\right)$ :

$$
\Phi\left(r=r_{0}, \chi\right)=\frac{1}{2} \sum_{m=-M / 2}^{M / 2}\left(\Phi_{m}+\Phi_{-m}^{*}\right) e^{i m \chi},
$$

having invoked the reality condition. The re-normalized field $\tilde{\Phi}\left(r_{0}, \chi\right)$ is then given by:

$$
\tilde{\Phi}\left(r_{0}, \chi\right)=\frac{A}{2} \sum_{m=-M / 2}^{M / 2}\left(\Phi_{m} e^{i \Delta \theta}+\Phi_{-m}^{*} e^{-i \Delta \theta}\right) e^{i m \chi}
$$

where the real amplitude $A$ and phase shift $\Delta \theta$ are adapted so that the complexe coefficient $\Phi_{m}$ of the dominant poloidal Fourier mode obtained from the two codes match. Following this procedure, the two electrostatic potentials are shown in Fig. 4, confirming the very close agreement.

The radial structure of the mode is also analyzed. In Fig. 6, the squared amplitude of the electrostatic potential from GENE and ORB5, averaged over the poloidal direction, is shown as a function of the minor radius $r$. A similar shape of the envelope is observed for the two simulation results. One notes however that the peaked positions slightly differ, which may also account for the differences in the real frequencies observed in Fig. 3. 


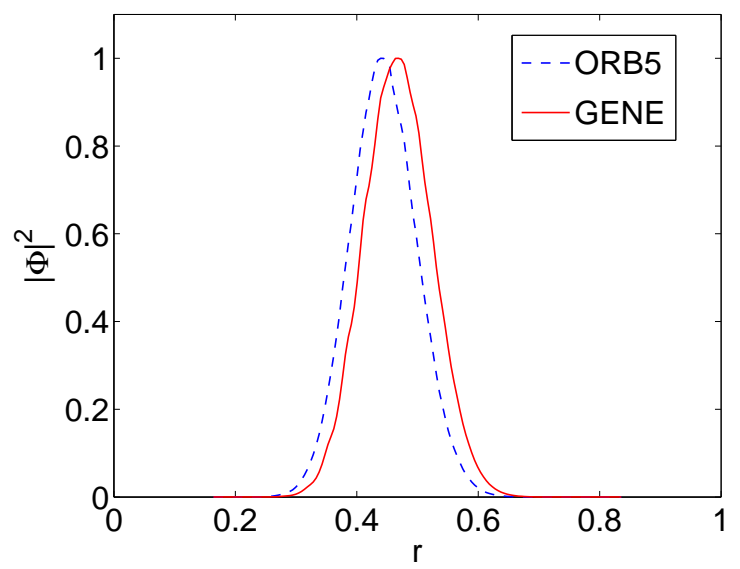

FIG. 6. (Color online) Radial profile of the poloidal averaged $|\Phi|^{2}$ for $k_{\theta} \rho_{i}=0.3$.

\section{ROSENBLUTH-HINTON TEST}

The Rosenbluth-Hinton test ${ }^{28}$ consists of computing the linear evolution of the zonal flow component $(n=0, m=0)$, where $m$ is the poloidal mode number, for an initial electrostatic perturbation $\Phi$. From the analytical resolution of the gyrokinetic equation for $n=0$, and local to a given magnetic surface, one expects to observe a damped oscillation of the Geodesic Acoustic Modes $(\mathrm{GAM})^{29}$ relaxing towards the zonal flow residual ${ }^{28}$. For a simplified set-up, in particular large aspect ratio and small $\rho^{*}=\rho_{i} / a$, the time evolution of the the zonal flow component can be written:

$$
\frac{E_{r}(t)}{E_{r}(0)}=\left(1-A_{R}\right) e^{-\gamma_{R} t} \cos \left(w_{g} t\right)+A_{R}
$$

where $E_{r}=-\partial\langle\Phi\rangle / \partial x$ is the radial perturbed electric field, $\langle\Phi\rangle$ being the flux-surface averaged potential and $\left(\omega_{G}, \gamma_{G}\right)$ the GAM frequency and damping rate respectively. The residual is

$$
A_{R}=\frac{1}{1+1.6 q^{2} / \sqrt{r / R}},
$$

with $r$ the minor radius of the considered magnetic surface, $R$ the major radius, $q$ the safety factor on the surface of interest. A correct prediction of this residual level is an important test for gyrokinetic codes, as zonal flows are identified to be a key saturation mechanism in turbulent regimes, in particular for ITG turbulence. Both GENE and ORB5 have already been successfully compared to analytical results ${ }^{20,22}$. We have observed, that in order to 
obtain such good quantitative agreement with the analytical predictions, it is necessary to use a constant or linear safety factor profile so as to be closer to the local assumptions considered for deriving Eq. (19). In the present benchmark study, the Rosenbluth-Hinton test is carried out assuming a more realistic quadratic safety factor profile and results from the two codes are therefore compared with each other instead of confronting them against the analytical relations. The physical parameters used in the following are:

$$
a / R=0.1, \quad q(r)=0.96+0.75(r / a)^{2}
$$

$\rho^{*}=\rho_{i} / a=1 / 160$, flat temperature and density profiles $\left(\kappa_{T i}=\kappa_{n i}=0\right)$, and an adiabatic electron response is again assumed.

The time evolution of the zonal flow component obtained with the two codes is shown in Fig. 7, for $r / a=0.3$. One observes very similar GAM damping rates and frequencies. The residual level predicted by the GENE code is $A_{R}=0.082$ which agrees within $10 \%$ with the ORB5 results, providing $A_{R}=0.074$. Note that, for the present parameters, the analytical prediction is $A_{R}=0.093$.

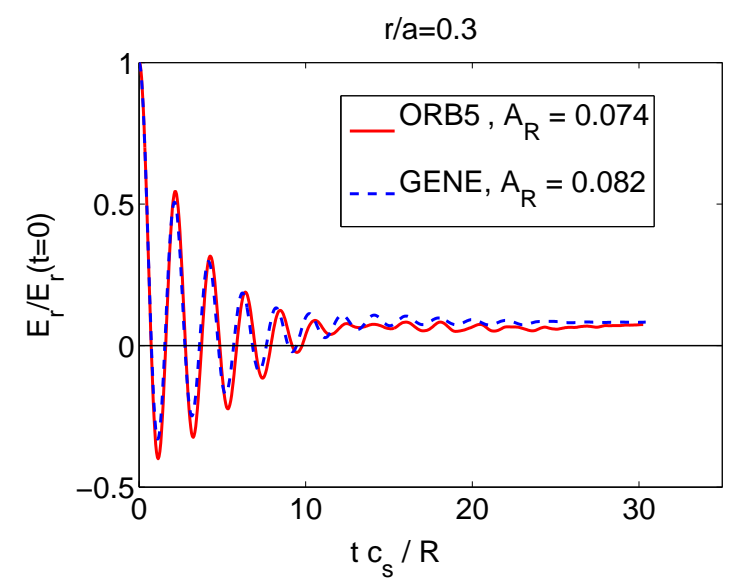

FIG. 7. (Color online) Time evolution of the normalized electric field at $r / a=0.3$, obtained with GENE and ORB5. 


\section{NONLINEAR RESULTS}

The nonlinear results in this section are obtained with Cyclone-like parameters, as in Sec. III, i.e. with $a / R=0.36, \rho^{*}=\rho_{i} / a=1 / 180$, and a safety factor profile:

$$
q(r)=0.86-0.16 r / a+2.52(r / a)^{2}
$$

For the GENE simulations, a radial domain of width $0.8 a$ centered at $r=r_{0}=0.5 a$ is used and a toroidal wedge corresponding to $1 / 3$ of the Tokamak is considered, such that one out of every three toroidal mode numbers is retained. The simulations are solved for a total of 32 toroidal modes, corresponding to a resolved spectrum ranging from $k_{\theta} \rho_{s}=0.048$ to 1.54. The box size is $l_{x} \times l_{y} \times l_{z} \times l_{v_{\|}} \times l_{\mu}=144 \rho_{s} \times 132 \rho_{s} \times 2 \pi \times 4 v_{t h, i} \times 16 T_{i 0} / B_{\text {ref }}$, with $v_{t h, i}=\sqrt{2 T_{i 0} / m_{i}}, T_{i 0}=T_{i}\left(r=r_{0}\right), r_{0}=0.5 a$ and $B_{\text {ref }}$ the magnetic field on axis. One also uses $\rho_{s}=c_{s} / \Omega_{i}$, with $c_{s}=\sqrt{T_{e 0} / m_{i}}, T_{e 0}=T\left(r=r_{0}\right), \Omega_{i}=e B_{0} / m_{i}$. The corresponding grid resolution is taken as $n_{x} \times n_{y} \times n_{z} \times n_{v_{\|}} \times n_{\mu}=150 \times 64 \times 16 \times 64 \times 16$. Dirichlet boundary conditions are used in the radial direction for both $\Phi$ and $\delta f$. In addition, damping regions are considered in the vicinity of the inner and outer radial boundary each corresponding to $5 \%$ of the total radial domain ${ }^{14}$.

Concerning the ORB5 results, the radial width of the domain centered at $r=r_{0}=0.5 a$ is $0.9 a$, and a toroidal wedge corresponding to $1 / 2$ of the Tokamak is considered, such that one out of every two toroidal mode numbers is retained. The simulations are carried out considering a total of 45 toroidal modes, corresponding to a resolved spectrum ranging from $k_{\theta} \rho_{s}=0.032$ to 1.45 , ensuring a similar largest resolved $k_{\theta}$ in the two codes. The number of markers is $320 \mathrm{M}$ and the grid resolution for the fields is $N_{s} \times N_{\chi} \times N_{\Phi}=128 \times 512 \times 256$. Free boundary conditions are considered for $\Phi$ at the inner edge and Dirichlet at the outer edge. Concerning the perturbed distribution function $(\delta f)$, the boundary conditions are such that any marker that leaves the domain at $\left(r_{\mathrm{bnd}}, \theta, \phi, v_{\|}, \mu\right)$ is re-injected at a symmetric position with respect to the equatorial mid-plane $\left(r_{\mathrm{bnd}},-\theta, \phi, v_{\|}, \mu\right)$. In an Eulerian description, this reads:

$$
\delta f\left(r_{\text {bnd }}, \theta, \phi, v_{\|}, \mu\right)=\delta f\left(r_{\text {bnd }},-\theta, \phi, v_{\|}, \mu\right)
$$

One notes that the following comparisons are carried out considering the standard mode of operation of the two codes, in particular different boundary conditions are retained. These differences should only marginally influence the physical results, and good agreement 
between the simulations thus justify a posteriori the implementation choices that have been considered in the two codes.

\section{A. Relaxation problem}

As a first comparison, nonlinear simulations without heat sources are presented. One considers here peaked logarithmic gradient profiles of ion temperature and density, as defined by Eq. (15), with $\kappa_{T i}=6.96, \kappa_{n}=2.23, \Delta T_{i}=0.3$ and $\Delta n=0.3$. The electron-ion temperature ratio is $\tau=T_{e} / T_{i}=1$ throughout the plasma.

The corresponding time evolutions of the nonlinear effective ion heat diffusivity $\chi_{i}=$

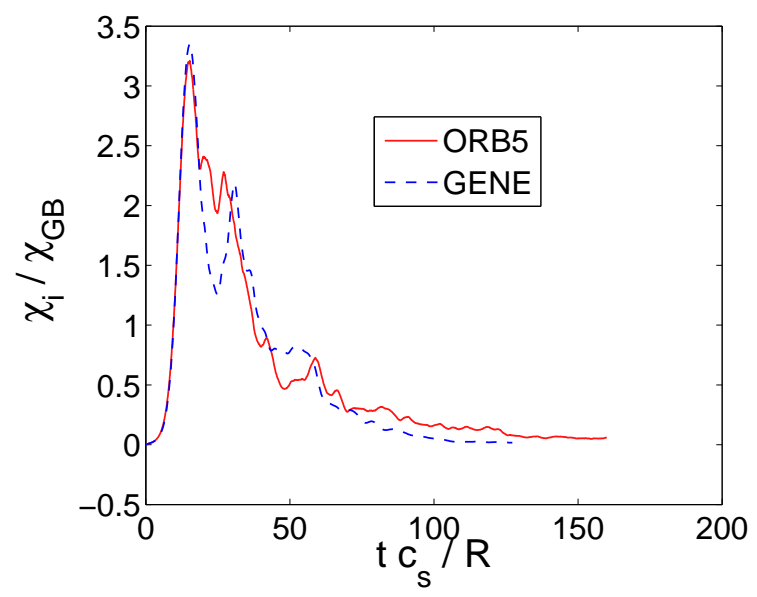

FIG. 8. (Color online) Time evolutions of the nonlinear heat diffusivity $\chi / \chi_{G B}\left(\chi_{G B}=\rho_{i}^{2} c_{s} / a\right)$ obtained with ORB5 and GENE for CBC parameters. Note the decrease of the heat diffusivity as no sources are included.

$\left\langle Q_{i}\right\rangle /\left\langle\left|\nabla T_{i}\right|\right\rangle$, where $\left\langle Q_{i}\right\rangle$ denotes a radial average between $r / a=0.4$ and 0.6 , obtained with ORB5 and GENE are shown in Fig. 8, and are given in gyro-Bohm units $\chi_{G B}=\rho_{s}^{2} c_{s} / a$. For this first nonlinear test, particular effort was made to start the simulations of both codes with exactly the same initial condition taken as:

$$
\delta f\left(x, k_{y}, z\right)=F_{k_{y}} \exp \left[-\frac{1}{2}\left(\frac{x}{\sigma_{x}}\right)^{2}-\frac{1}{2}\left(\frac{k_{y}}{\sigma_{y}}\right)^{2}-\frac{1}{2}\left(\frac{z}{\sigma_{z}}\right)^{2}\right] f_{0}
$$

with $F_{k_{y}}=1$ for $k_{y} \neq 0$ and $F_{k_{y}}=10^{-2}$ for $k_{y}=0$. The remaining widths of the initial gaussian perturbation in the $\mathrm{x}, \mathrm{y}, \mathrm{z}$ directions are respectively given by $\sigma_{x}=10 \rho_{i}, \sigma_{y} \rho_{i}=$ 
0.385 and $\sigma_{z}=\pi / 4$. The linear phases $\left(t c_{s} / R \lesssim 20\right)$ from the two simulations are therefore, as expected, essentially identical. Moreover, a very similar amplitude of the first burst is observed in both GENE and ORB5 simulations. The subsequent evolution is stochastic and the two time traces thus inevitably come to differ and can only be compared statistically (in terms of mean value, variance, etc $)^{30}$. Since no sources are considered here, the ion temperature profile rapidly relaxes towards its nonlinear marginal value, which leads to a decrease of the turbulence and the resulting heat diffusivity. Although a good qualitative agreement is reached between the two codes, it is difficult to evaluate precisely the differences between the two time traces after the first burst as no steady state is reached and therefore insufficient time to acquire statistically significant estimates of the heat flux at a given gradient value.

\section{B. Nonlinear results with sources}

In order to carry out more quantitative nonlinear comparisons between the two codes, the Krook-type heat sources given by Eqs. (10)-(11) are now switched on for the ions, with $\gamma_{h} R / c_{s}=0.035$. This value is chosen about ten times smaller than the typical linear growth rate, so that the time scale on which the heat source affects the temperature profile is an order of magnitude smaller the linear phase. For these simulations, logarithmic gradient profiles for the characteristic time of turbulent eddy growth, estimated by $\gamma_{m} a x R / c_{s}=0.27$ according to Fig. 2. For these simulations, logarithmic gradient profiles for initial density and temperature are used according to the following functional form (A stands for $n$ or $T_{i}$ ):

$$
R \frac{d \ln A}{d r}=-\kappa_{A}\left[1-\cosh ^{-2}\left(\frac{r-\left(r_{0}-\Delta r / 2\right)}{a \Delta A}\right)-\cosh ^{-2}\left(\frac{r-\left(r_{0}+\Delta r / 2\right)}{a \Delta A}\right)\right]
$$

taken for $r \in\left[r_{0}-\Delta r / 2, r_{0}+\Delta r / 2\right]$ and zero outside. These gradient profiles are flatter than the ones considered in Sec. VA. The different constant parameters are set to $\kappa_{T i}=7.1$, $\kappa_{n}=2.2, \Delta r=0.8 a$ and $\Delta T_{i}=\Delta n=0.04$. The corresponding profile and logarithmic gradient profile for the ion temperature, radially average in the interval $0.4 \leq r / a \leq 0.6$ are shown in Fig. 9.

Using these initial profiles, the time evolution of the heat diffusivity and normalized logarithmic gradient of the total temperature $T_{i \text {,tot }}=T_{i 0}+\delta T_{i}$, where $\delta T_{i}$ is the temperature profile variation related to $\delta f$, are shown in Figs. 10 and 12, together with their running 


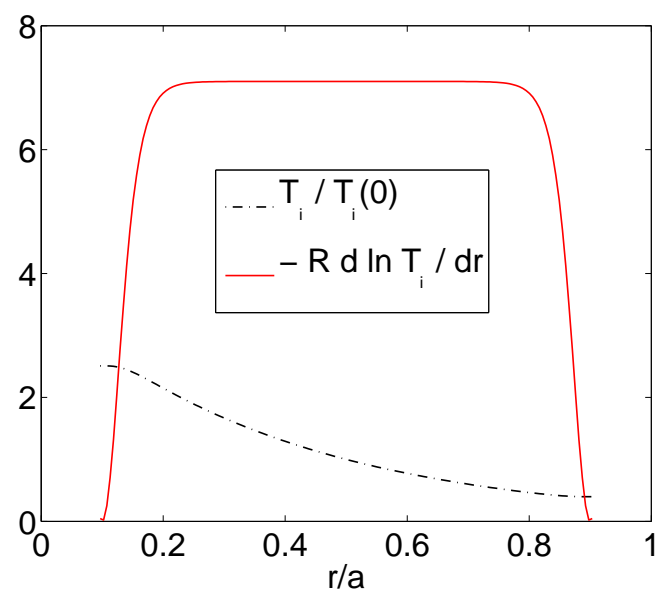

FIG. 9. (Color online) Temperature and corresponding logarithmic gradient profile for $\kappa_{T i}=7.1$, $\Delta r=0.8 a$ and $\Delta T_{i}=0.04$ [see Eq. (24)].

time-average starting at $t_{0}=150 R / c_{s}$, defined for a quantity $A$ as:

$$
A_{\mathrm{av}}(t)=\frac{1}{t-t_{0}} \int_{t_{0}}^{t} A(t) d t, \quad \text { for } \quad t>t_{0} .
$$

As opposed to the case with no sources, a quasi-steady state is reached here, illustrating the advantage of using such a heat source for this code comparison as it enables to acquire statistically relevant estimates of the turbulent regime. The averaged heat diffusivity over the time interval $t c_{s} / R=[180,420]$, including approximately 10 bursts, is $\chi_{i} / \chi_{G B}=1.95$ for GENE and $\chi_{i} / \chi_{G B}=1.76$ for ORB5, i.e. a relative difference of about $10 \%$. This small deviation is of the same order as between the linear results shown earlier and may again be partly accounted for by the different treatment of the $\nabla p_{0}$ term as well as the different field solvers considered, which is only second order accurate in $k_{\perp} \rho_{i}$ in ORB5 while all orders are retained in GENE. Moreover, it should be noted that studies carried out with ORB5 have shown that the heat diffusivity computed with different initial conditions could vary within $\pm 15 \%^{27,30}$. The present agreement is thus considered very satisfying.

In order to further compare the two simulations the Fourier spectra of the space and time averaged field energy $\langle\delta n \Phi\rangle_{k}$ are shown in Fig. 11 as function of the poloidal wave number $k_{\theta} \rho_{i}$. A good agreement is here observed, in particular a similar decay, with near algebraic scaling is shown in both cases in the region $0.3<k_{\theta} \rho_{i}<1.2$. The measured slope in this 


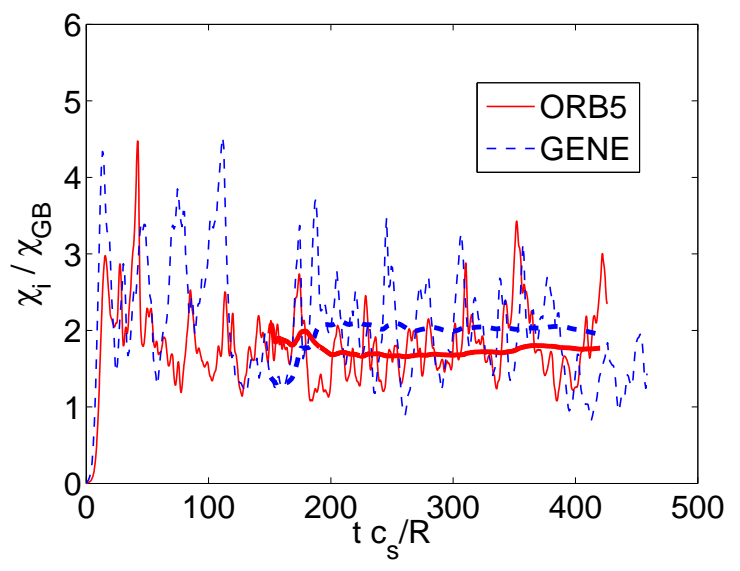

FIG. 10. (Color online) Time evolution of the ion heat diffusivity $\chi_{i}$ in units of $\chi_{G B}=c_{s} \rho_{s} / a^{2}$. These quantities have been obtained by radial averaging of the heat flux and ion temperature gradient over the range $r / a=[0.4,0.6]$. The bold lines represent the running time-average starting from $t_{0}=150 R / c_{s}$.

region is $k \simeq-3.5$. The effects of the $k_{\perp} \rho_{i}$ approximations in ORB5 field solver appears to be only significant for $k_{\theta} \rho_{i}>1.2$ where one clearly sees an important decrease of the mode amplitudes. This particular approximation thus has only a minor influence on the present results. Considering the region $0.15<k_{\theta} \rho_{i}<0.3$ which mostly contributes to the transport, one observes that the mode amplitudes obtained with ORB5 are smaller than those obtained with GENE, consistent with $\chi_{i}$ values. We thus conclude that most differences on the total heat diffusivity between the two codes result from contributions in this part of the spectrum.

Focusing now on the time evolution of the logarithmic gradient of the ion temperature in Fig. 12, one observes that the quasi-steady state values, $R / L_{T i}=6.7$ for GENE and $R / L_{T i}=6.8$ for ORB5, are smaller than the initial value $R / L_{T i}=7.1$. This can be explained by the value of the rate $\gamma_{h}$ used in the heat source operator, which is chosen about 10 times lower than the typical growth rate. The quasi-steady state value of the total temperature gradient therefore reflects a partial relaxation corresponding to an equilibrium between the turbulent transport which tends to flatten the temperature profile and the Krook-type heat source which restores the temperature profile towards the initial background profile. 


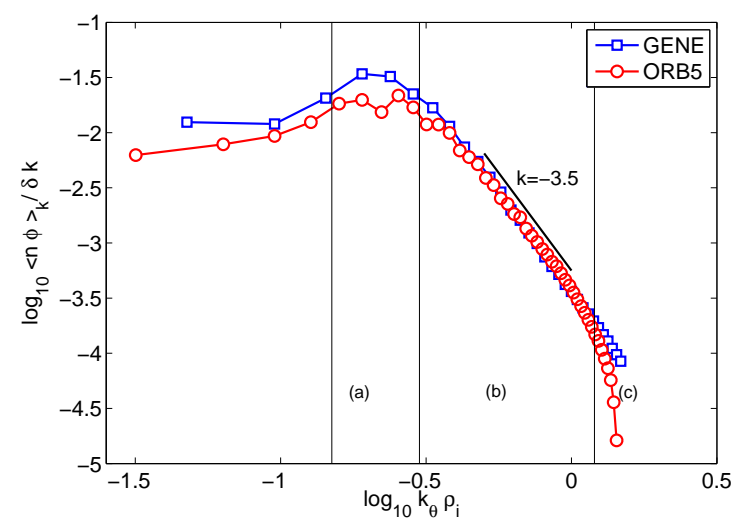

FIG. 11. (Color online) Time and spacial averaged spectrum of $\langle\delta n \Phi\rangle_{k}$, shown in logarithmic scales. The averages are taken in the $t c_{s} / R=[150,400]$ interval and considering the full spacial domain. The spectrum is defined such that $\sum_{k} \Delta k\langle\delta n \Phi\rangle_{k}=\langle\delta n \Phi\rangle$, and the perturbed density and electrostatic potential are respectively normalized to $T_{i} \rho_{i} /(e R)$ and $n_{0} \rho_{i} / R$. Regions corresponding to $0.15<k_{\theta} \rho_{i}<0.3,0.3<k_{\theta} \rho_{i}<1.2$ and $k_{\theta} \rho_{i}>1.2$ have been respectively labelled (a),(b) and (c). One notes an overall good agreement of the spectra and in particular very similar inertial-type range with near algebraic scaling $(k \simeq-3.5)$ are observed in region $(b)$ in both cases.

As already mentioned in section IV, the zonal flow component $k_{y}=0$ (i.e. $n=0$ ) plays a key role for the nonlinear saturation in ITG regime, and an accurate description of its structure is therefore of particular importance when comparing nonlinear simulations. In Fig. 13, a two-dimensional representation of the normalized flux-surface averaged radial electric field $E_{r}(t, x)$ is shown, defined as:

$$
\tilde{E}_{r}(t, r)=\frac{\rho_{s}^{2} e}{R T_{e}} \frac{\partial\langle\Phi\rangle}{\partial r},
$$

with $\Phi$ the electrostatic potential and \langle\rangle the flux-surface average. When comparing GENE and ORB5 results in the inner part of the simulation domain $(r / a=[0.3,0.7])$, one observes similar small-scale avalanche-like structures ${ }^{31}$ in both cases. In order to compare quantitatively the radial structure of the flux-surface averaged electric fields, the time-average of $E_{r}(t, x)$ over the interval $t c_{s} / R=[380,420]$ is in addition shown in Fig. 14. The radial profiles of the electric field $E_{r}$ present in both cases multiple local extrema at similar positions. One notes, however, that the absolute amplitudes of the fields are different, which can in fact be explained by the different radial boundary conditions used in the two codes. 


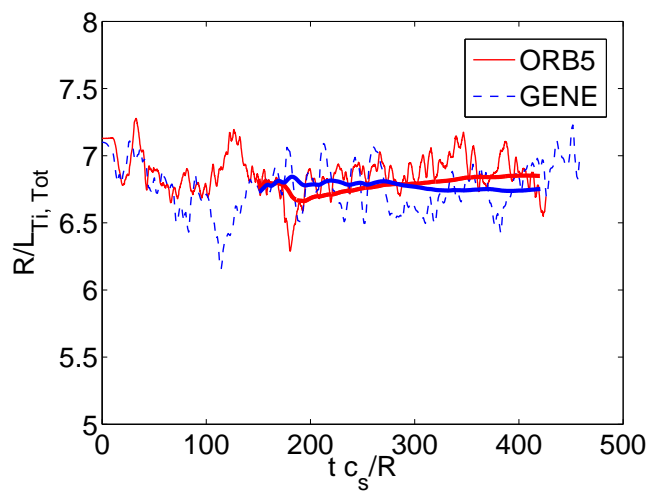

FIG. 12. (Color online) Time evolution of the normalized logarithmic gradient $R_{L_{T i \text { Tot }}}$ of the total ion temperature $T_{i}=T_{0 i}+T_{1 i}$ for CBC like parameters, obtained by radial averaging over the range $r / a=[0.4,0.6]$. The bold lines represent the running time-average starting from $t_{0}=150 R / c_{s}$.
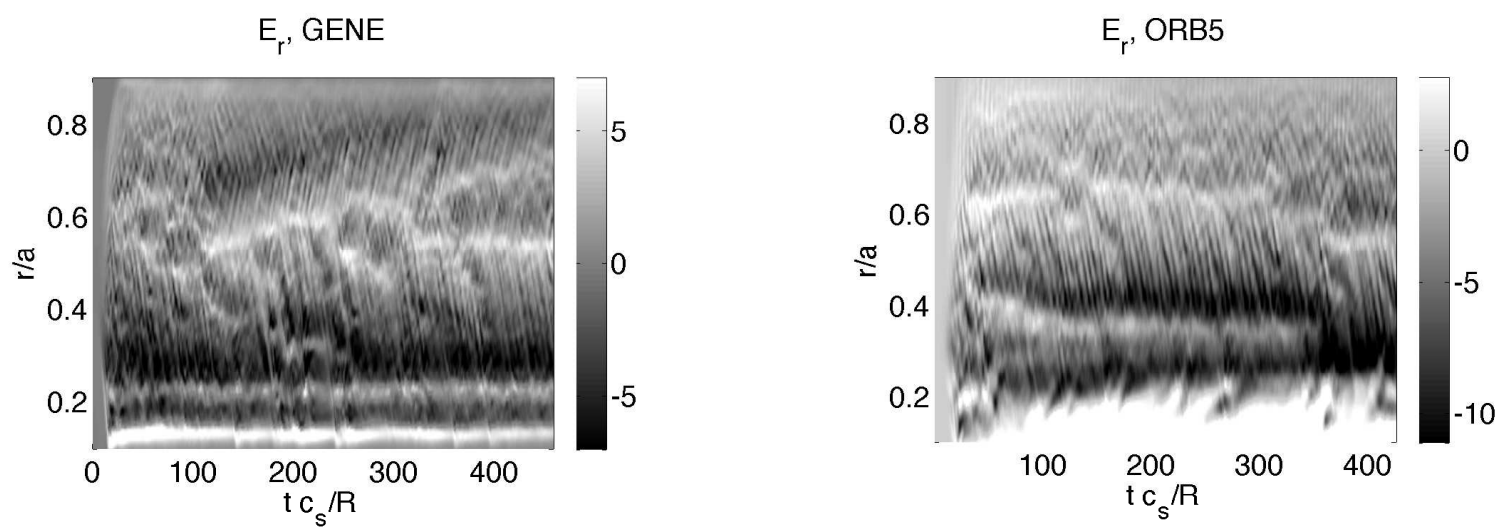

FIG. 13. Normalized flux-surface averaged electric field $E_{x}$ obtained with GENE and ORB5 as a function of the radial coordinate $x$ and time $t$.

The influence of zonal flow on microturbulence results from its capability to shear the radial coherent turbulent structures ${ }^{32}$. This effect depends on the shearing rate $\omega_{E \times B}$, which is proportional to the first radial derivative of the electric field (i.e. second derivative of the electrostatic potential):

$$
\omega_{E \times B} \propto \frac{d E_{r}}{d r} \propto \frac{d^{2}\langle\Phi\rangle}{d r^{2}} .
$$

By comparing in Fig. 15 the radial profiles of $d E_{r} / d r$, one observes a very good quantitative agreement between the two codes. This further explains the similar values obtained for the heat diffusivity and shows that the choice of boundary conditions in the radial direction for 


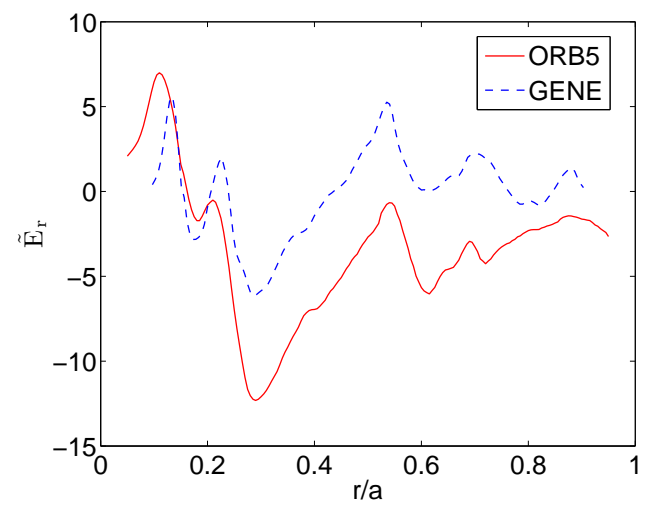

FIG. 14. (Color online) Radial profile of the normalized flux-surface averaged electric field $E_{r}$, further averaged over the time interval $t c_{s} / R=[380420]$, obtained with GENE and ORB5.

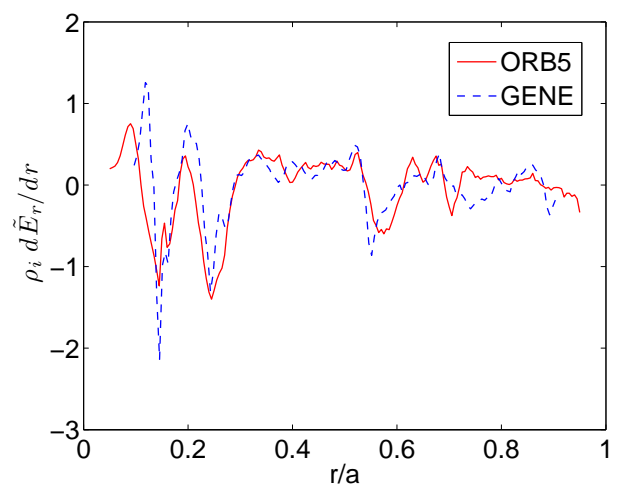

FIG. 15. (Color online) Radial profile of the derivative $d E_{r} / d r$, averaged over the time interval $t c_{s} / R=[380420]$.

$\Phi$ seems to have little effect on the physical simulation results.

\section{CONCLUSIONS}

Several linear and nonlinear benchmarks between the global gyrokinetic codes ORB5 and GENE have been carried out in the present work. In the linear regime, a good agreement 
was reached between the two codes for Cyclone like parameters ${ }^{16}$ concerning the growth rates and real frequencies. Some of the remaining discrepancies in the growth rates could be accounted for by the different treatment in the two codes of the pressure correction term appearing in the magnetic curvature drift. In addition, the electrostatic potential fields have been compared and very similar mode structures were observed. Considering nonlinear results computed with identical initial conditions, excellent agreement has been observed even up to the first burst, where nonlinear effects clearly affect the simulation, has been observed. A Krook-type heat source was then introduced, allowing for the first time to compare global gyrokinetic codes in quasi-steady state and to obtain statistically relevant estimates of the averaged heat diffusivity and effective temperature gradient. It was shown that a level of agreement within $10 \%$ could be reached under such conditions. In addition, investigations of the zonal flow structure were carried out by comparing the flux-surface averaged radial electric field. A similar overall radial structure of the radial electric fields was observed, although the amplitudes were found to differ as a result of the different radial boundary conditions used in the two codes. More importantly however, it was shown that the derivative of the radial electric field, which is directly related to the shearing rate, agree very well.

The presented comparisons were pushed to a new level of detail and thus represent a useful contribution to the ongoing general effort for benchmarking gyrokinetic codes. Particular attention was given to providing all necessary information required for reproducing these simulations with other codes.

The high level of agreement between a global Eulerian and a global Lagrangian PIC code obtained in the present work has been further confirmed in studies of finite size scaling of turbulent transport ${ }^{33}$.

\section{ACKNOWLEDGMENTS}

This work has been partly supported by the Swiss National Science Foundation. The simulations have been run on the HPC-FF computer in Jülich, Germany. 


\section{REFERENCES}

${ }^{1}$ E. A. Frieman and L. Chen, Phys. Fluids, 25, 502 (1982).

${ }^{2}$ T. S. Hahm, Phys. Fluids, 31, 2670 (1988).

${ }^{3}$ X. Garbet, Y. Idomura, L. Villard, and T. Watanabe, Nuclear Fusion, 50, 043002 (2010).

${ }^{4}$ S. E. Parker and W. W. Lee, Phys. Fluids, B 5, 77 (1993).

${ }^{5}$ M. Kotschenreuther, G. Rewoldt, and W. Tang, Comput. Phys. Comm., 88, 128 (1995).

${ }^{6}$ A. M. Dimits, T. J. Williams, J. A. Byers, and B. I. Cohen, Phys. Rev. Lett., 77, 71 (1996).

${ }^{7}$ F. Jenko, W. Dorland, M. Kotschenreuther, and B. N. Rogers, Phys. Plasmas, 7, 1904 (2000).

${ }^{8}$ A. Peeters and D. Strintzi, Phys. Plasmas, 11, 3748 (2004).

${ }^{9}$ Z. Lin, T. Hahm, W. W. Lee, W. M. Tang, and R. White, Science, 281, 1835 (1998).

${ }^{10}$ R. Sydora, Phys. Scripta, 52, 474 (1995).

${ }^{11}$ T. Tran, K. Appert, M. Fivaz, G. Jost, J. Vaclavik, and L. Villard, Proceedings of Invited Papers, Joint Varenna - Lausanne Int. Workshop on Theory of Fusion Plasmas, Varenna, Italy, 55 (1998).

${ }^{12}$ Y. Idomura, S. Tokuda, and Y. Kishimoto, Nucl. Fusion, 43, 234 (2003).

${ }^{13}$ V. Grandgirard, Y. Sarazin, P. Angelino, A. Bottino, N. Crouseilles, G. Darmet, G. DifPradalier, X. Garbet, P. Ghendrih, S. Jolliet, G. Latu, E. Sonnendrucker, and L. Villard, Plasma Phys. Control. Fusion, 49, B173 (2007).

${ }^{14}$ T. Görler, X. Lapillonne, S. Brunner, T. Dannert, F. Jenko, F. Merz, and D. Told, "The global version of the gyrokinetic turbulence code GENE", submited to J. Comput. Phys. ${ }^{15}$ M. A. Beer, S. C. Cowley, and G. W. Hammett, Phys. Plasmas, 2, 2687 (1995).

${ }^{16}$ A. M. Dimits, G. Bateman, M. A. Beer, B. I. Cohen, W. Dorland, G. W. Hammett, C. Kim, J. E. Kinsey, M. Kotschenreuther, A. H. Kritz, L. L. Lao, J. Mandrekas, W. M. Nevins, S. E. Parker, A. J. Redd, D. E. Shumaker, R. Sydora, and J. Weiland, Phys. Plasmas, 7, 969 (2000).

${ }^{17}$ W. M. Nevins, J. Candy, S. Cowley, T. Dannert, A. Dimits, W. Dorland, C. Estrada-Mila, G. W. Hammett, F. Jenko, M. J. Pueschel, and D. E. Shumaker, Physics of Plasmas, 13, 122306 (2006). 
${ }^{18}$ W. M. Nevins, S. E. Parker, Y. Chen, J. Candy, A. Dimits, W. Dorland, G. W. Hammett, and F. Jenko, Physics of Plasmas, 14, 084501 (2007).

${ }^{19}$ G. L. Falchetto, B. D. Scott, P. Angelino, A. Bottino, T. Dannert, V. Grandgirard, S. Janhunen, F. Jenko, S. Jolliet, A. Kendl, B. F. McMillan, V. Naulin, A. H. Nielsen, M. Ottaviani, A. G. Peeters, M. J. Pueschel, D. Reiser, T. T. Ribeiro, and M. Romanelli, Plasma Phys. Control. Fusion, 50, 124015 (2008).

${ }^{20}$ T. Görler, Ph.D. thesis, Universität Ulm (2009).

${ }^{21}$ X. Lapillonne, Ph.D. thesis, Ecole Polytechnique Fédérale de Lausanne (2010).

${ }^{22}$ S. Jolliet, A. Bottino, P. Angelino, R. Hatzky, T. M. Tran, B. F. Mcmillan, O. Sauter, Y. Idomura, and L. Villard, Comput. Phys. Comm., 177, 409 (2007).

${ }^{23}$ A. Bottino, B. Scott, S. Brunner, B. McMillan, L. Villard, S. Jolliet, R. Hatzky, and A. G. Peeters, IEEE Trans. Plasma Sci., 38, 5535196 (2010).

${ }^{24}$ P. W. Terry, M. Greenwald, J.-N. Leboeuf, G. R. McKee, D. R. Mikkelsen, W. M. Nevins, D. E. Newman, D. P. Stotler, Task Group on Validation and Verification, U.S. Burning Plasma Organization, and U.S. Transport Task Force, Phys. Plasmas, 15, 062503 (2008). ${ }^{25}$ P. Angelino, A. Bottino, R. Hatzky, S. Jolliet, O. Sauter, T. M. Tran, and L. Villard, Physics of Plasmas, 13, 052304 (2006).

${ }^{26}$ X. Lapillonne, S. Brunner, T. Dannert, S. Jolliet, A. Marinoni, L. Villard, T. Görler, F. Jenko, and F. Merz, Phys. Plasmas, 16, 032308 (2009).

${ }^{27}$ B. F. McMillan, S. Jolliet, T. M. Tran, L. Villard, A. Bottino, and P. Angelino, Phys. Plasmas, 15, 052308 (2008).

${ }^{28}$ M. N. Rosenbluth and F. L. Hinton, Phys. Rev. Lett., 80, 724 (1998).

${ }^{29}$ N. Winsor, J. L. Johnson, and J. M. Dawson, Physics of Fluids, 11, 2448 (1968).

${ }^{30}$ L. Villard, A. Bottino, S. Brunner, A. Casati, J. Chowdhury, T. Dannert, R. Ganesh, X. Garbet, T. Görler, V. Grandgirard, R. Hatzky, Y. Idomura, F. Jenko, S. Jolliet, S. K. Aghdam, X. Lapillonne, G. Latu, B. F. McMillan, F. Merz, Y. Sarazin, T. M. Tran, and Y. T. Vernay, "Gyrokinetic simulations of turbulent transport: size scaling and chaotic behaviour," Plasma Phys. Control. Fusion 52 2010, accepted, to appear.

${ }^{31}$ B. F. McMillan, S. Jolliet, T. M. Tran, L. Villard, A. Bottino, and P. Angelino, Phys. Plasmas, 16, 022310 (2009).

${ }^{32}$ P. H. Diamond, S.-I. Itoh, K. Itoh, and T. S. Hahm, Plasma Phys. Control. Fusion, 47, R35 (2005). 
${ }^{33}$ B. F. McMillan, X. Lapillonne, S. Jolliet, L. Villard, S. Brunner, A. Bottino, T. Görler, and F. Jenko, Phys. Rev. Let., 105, 1555001 (2010). 